\title{
Correlation between Serum Levels of Sympathetic Nerve Activity Markers and Sleep Quality and Cognitive Function in Patients with Chronic Insomnia Disorder
}

\author{
Ting $\mathrm{Hu}^{1,2}$, Xuan Song ${ }^{1,3}$, Yi-Jun $\mathrm{Ge}^{1}$, Ping Zhang ${ }^{1}$, Xue-Yan $\mathrm{Li}^{1}$, Xiao-Yi Kong ${ }^{1}$ and \\ Gui-Hai Chen ${ }^{1, *}$
}

${ }^{1}$ Department of Sleep Disorders, the Affiliated Chaohu Hospital of Anhui Medical University, Hefei (Chaohu), China

${ }^{2}$ Department of General Practice, Anqing Municipal Hospital of Anhui Medical University, Anqing, China

${ }^{3}$ Department of General Practice, Xuancheng Municipal Hospital, Xuancheng, China

\begin{abstract}
Objectives: To explore the changes of the serum levels of copeptin and a-amylase and the correlations with sleep quality and cognition function in the patients with chronic insomnia disorder (CID).

Methods: Fifty-seven CID patients and thirty healthy controls were enrolled continuously. Pittsburgh Sleep Quality Index (PSQI), polysomnography (PSG) and Pre-Sleep Arousal Scale (PSAS) were used to assess the insomnia severity and cognitive and somatic manifestations of arousal experienced at bedtime. Montreal Cognitive Assessment scale (MoCA) and Nine-Box Maze were used to respectively assess general cognition and memories. The serum levels of copeptin and $\alpha$-amylase were detected using Enzyme-Linked ImmunoSorbent Assay.

Results: Compared to the controls, the CID patients had increased PSQI and PSAS scores ( $Z=-7.678$ and -7.350 ; $P S<0.001)$, decreased MoCA score $(t=-4.625, P<0.001)$, increased numbers of errors in the object working, spatial working and object recognition $(Z=-2.099,-3.935$ and $-2.266 ; P s<0.05)$ memories, and elevated serum levels of copeptin and $\alpha$-amylase $(t=5.414$ and 5.597, $P<0.001)$. In the CID patients, the level of copeptin positively correlated with PSQI and PSAS scores ( $r=0.338$ and $0.316 ; P s<0.05)$, and PSG sleep latency, wake time and N1\% $(r=0.324,0.325$ and $0.278, P s<0.05)$, and negatively correlated with PSG N $2 \%(r=-0.279, P s<0.05)$. Alpha-amylase was positively correlated with waking numbers in PSG $(r=0.293, P<0.05)$. Multiple linear regression analysis showed that copeptin level affected PSQI score and PSG sleep latency $(P<0.05)$.
\end{abstract}

Conclusions: The serum levels of copeptin and $\alpha$-amylase elevated in the CID patients, and the serum levels of copeptin may be associated with the poor sleep quality, especially in the individuals of initial sleep difficulties.

Keywords: a-amylase, Cognition, Copeptin, Insomnia.

\section{INTRODUCTION}

Chronic insomnia disorder (CID) is a patientreported problem characterized by difficulty falling asleep or difficulty maintaining sleep, i.e., frequent awakenings, difficulty returning to sleep after awakenings, or awakening too early with inability to return to sleep [1]. It is the most prevalent sleep disorder, affecting approximately $10 \%$ of the population worldwide [2]. CID is associated with higher levels of functional impairment (such as fatigue and irritability), higher frequency of psychiatric disorder, and a greater prevalence of chronic illness, contributing to an increased frequency of medical visits and missed work [3]. Even though the burden of CID on both individuals and society is significant, understanding the course of CID is difficult. In part, its complex psychoneurobiology not yet fully understood is the main reason.

"Address correspondence to this author at $t$ Department of Sleep Disorders, the Affiliated Chaohu Hospital of Anhui Medical University, Hefei (Chaohu), China; Tel: 13956094425; E-mail: doctorcgh@163.com
Although CID is considered a sleep disorder, its pathophysiology suggests hyperarousal during sleep and wakefulness [3]. Through "3P" model, psychophysiological model and neurocognitive model, experts and scholars have emphasized the close relationship between over-arousal and insomnia. Hyperarousal is a key component in all modern etiological models of CID, which considered that insomnia is the result of over-activation of central and peripheral multiple systems, including over-activation of hypothalamic-pituitary-adrenal (HPA) axis and sympathetic nervous system (SNS) [4, 5]. Modern neurobiological studies have found that insomnia patients have excessive arousal in autonomic nervous system, neuroendocrine system and neuroimmune system at night and during the day [6]. Previous studies paid more attention to the hyperfunction of the HPA axis in CID patients and found that corticotropinreleasing hormone and cortisol levels increased in these patients $[7,8]$. It is known that sympathetic nerve activation can also enhance HPA axis activity through 
arginine vasopressin (AVP). The physiological functions of AVP are homeostasis of fluid balance, vascular tonus and regulation of the endocrine stress response, but the AVP level (representing sympathetic nerve activity level) in CID patients has not been explored. Because of the short half-life, AVP in the blood is not easy to measure [9]. Copeptin and AVP derive from the same precursor molecule. Due to the equimolar secretion, copeptin responds as rapidly as AVP to osmotic, hemodynamic and unspecific stressrelated stimuli and both peptides show a very strong correlation [10]. Since copeptin, in contrast to AVP, can easily be measured with a sandwich immunoassay, it can indirectly indicate the amount of AVP in the circulation [11]. Copeptin has emerged as a useful measure in different diseases. On the other hand, alpha-amylase has been proposed as a sensitive biomarker for stress-related changes in the body that reflect the activity of the SNS [12], but the alphaamylase level in CID patients has not been explored.

In this study, we intend to explore the changes in sympathetic nerve activity index such as copeptin and a-amylase levels and their relationship with sleep quality and cognitive function in CID patients.

\section{MATERIALS AND METHODS}

\subsection{Subjects}

Patients with CID who were diagnosed and treated in Chaohu Hospital affiliated to Anhui Medical University from September 1, 2018, to May 31, 2019, were collected continuously. Inclusion criteria: Patients should meet the diagnostic criteria of chronic insomnia in International Classification of Sleep Disorders, Third Edition (ICSD-3) [13], aged 18 65 years old, with primary school education or above, and no understanding disorder. Chinese Pittsburgh Sleep Quality Index (PSQI) [14] total score>7, 17-term Hamilton Depression Scale (HAMD17) [15] total score<17. Exclusion criteria: (1) Patients who have taken any antidepressants, sedatives, and hypnotics in recent two weeks; (2) Patients with a history of alcoholism, serious endocrine diseases (such as liver disease, hyperthyroidism, pituitary tumor, etc.), and other diseases affecting amylase secretion (such as chronic pancreatitis, salivary gland diseases, etc.); (3) Pregnant or nursing patients; (4) Patients with vision and hearing impairment who cannot cooperate with the completion of the test; (5) Polysomnography (PSG) suggested that patients with sleep apnea index $<15$ times/h. Eventually, 57 patients with CID and 30 healthy people (control group, who had a physical examination in our hospital at the same time as CID patients, had no chief complaint of insomnia and related medical history, and both PSQI score and HAMD-17 score were $<7$ ) were included. This study was a cross-sectional study, approved by the Ethics Committee of Chaohu Medical College of Anhui Medical University (No.201805-kyxm-01), and all subjects and their families signed the informed consent form.

\subsection{General Data Collection}

The gender, age, education level, and disease course of subjects were collected. HAMD-17 was used to evaluate the depression status, and the method of the MINI-International Neuropsychiatric Interview (MINI) was used to exclude patients with definite depression symptoms. The Pre-Sleep Arousal Scale (PSAS) [16] was used to evaluate individuals' cognitive and physiological arousal before sleeping. PSAS consists of 16 questions, with a total score of 16 80, using the method of 1 5 points from "no feeling at all" to "extremely strong feeling." The higher the score is, the higher the degree of cognitive or physiological awakening is.

\subsection{Sleep Quality Evaluation}

PSQI was used to evaluate all subjects' subjective sleep quality in the last month. PSG (Compumedics Siesta 802, Compumedics, Australia) was also used to evaluate the CID group's objective sleep status. PSG examination was performed according to the standard of The AASM Manual for the Scoring of Sleep and Associated Events Rules, Terminology and Technical Specifications (2007 Edition). The analyzed indicators contained total sleep time, sleep latency, sleep efficiency, awakening times, awakening time after sleep, and the percentages of rapid eye movement (REM) sleep and non-REM sleep in stages 1,2, and 3 $(\mathrm{N} 1, \mathrm{~N} 2$, and $\mathrm{N} 3)$ in the total sleep time (REM\%, and $\mathrm{N} 1 \% \sim \mathrm{N} 3 \%)$.

\subsection{Cognitive Function Assessment}

All subjects were assessed with the Beijing-Chinese Montreal Cognitive Assessment (MoCA-C) scale [17]. According to the nine-box maze test [18] described by our group, the subjects' multiple memory functions were evaluated, including object/space reference memory, object/space working memory and object recognition memory, and the number of errors was 
recorded. The larger the number of errors is, the worse the memory is.

\subsection{Serological Index Detection}

From 8: 00 to 9: 00 the next morning after PSG monitoring, 2 3 $\mathrm{ml}$ of venous blood was taken (fasting before blood collection, avoiding strenuous exercise and mental stress). After standing for $30 \mathrm{~min}$, the serum was centrifuged at a speed of $3000 \mathrm{r} / \mathrm{min}$ for 5 min with a centrifugal radius of $15 \mathrm{~cm}$. The extracted serum was stored in a refrigerator at $-80^{\circ} \mathrm{C}$. ELISA method was used to determine the content of copeptin, and thiobarbituric acid (TBA method) was used to determine the content of $\alpha$-amylase. Specific test steps are strictly carried out regarding the operation flow of the Kit.

\subsection{Statistical Analysis}

SPSS 24.0 statistical software was used to statistically process the data. The measurement data conforming to normal distribution was expressed by the mean standard deviation (M $\pm \mathrm{SD})$, and the comparison between groups adopted the T-test of group design. The measurement data of non-normal distribution was expressed by the median (quartiles) [M (P25, P75)], and the rank-sum test (Mann-Whitney $U$ test) of group design was used for comparison between groups. Counting data was expressed by the number of cases and percentage, and the comparison between groups adopts $x^{2}$ test. Spearman analysis was used to analyze the correlation between serum, copeptin, and $\alpha$ amylase levels and clinical parameters in the CID group, and partial correlation analysis was completed after further controlling confounding factors (age, sex, education, and depression). Multivariate linear regression analysis was used to analyze the correlation between copeptins and variables. $P \leq 0.05$ as the difference was statistically significant.

\section{RESULTS}

\subsection{Clinical Baseline Data}

As shown in Table 1, there was no significant difference in gender, age, and education level between

Table 1: General Data, Serological Indexes and Sleep Parameters in Subjects

\begin{tabular}{|c|c|c|c|c|}
\hline Items & CID & Controls & Values & $P$-Values \\
\hline Male/Female(n) & $20 / 37$ & $12 / 18$ & $X^{2}=0.204$ & 0.652 \\
\hline Age (yr, M $\pm S D)$ & $50.1 \pm 9.7$ & $45.4 \pm 13.2$ & $t=1.889$ & 0.062 \\
\hline Years of Education [yr, M(P25, P75)] & $9.0(7.0,11.0)$ & $10.0(6.0,12.0)$ & $Z=-0.257$ & 0.797 \\
\hline 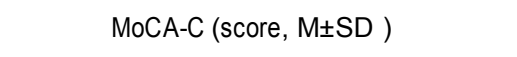 & $24.1 \pm 2.5$ & $26.7 \pm 1.9$ & $t=-4.625$ & 0 \\
\hline \multicolumn{5}{|l|}{ Serological index $(M \pm S D)$} \\
\hline Copeptin (pg/ml) & $35.1 \pm 19.9$ & $14.8 \pm 6.9$ & $t=5.414$ & 0 \\
\hline a-amylase (U/L) & $990.1 \pm 193.7$ & $728.9 \pm 230.5$ & $t=5.597$ & 0 \\
\hline \multicolumn{5}{|l|}{ Sleep awakening parameters } \\
\hline PSQI [score, M(P25, P75)] & $16.0(15.0,17.0)$ & $4.0(2.8,6.0)$ & $Z=-7.678$ & 0 \\
\hline PSAS [score, M(P25, P75)] & $33.0(30.0,37.5)$ & $17.0(6.0,18.5)$ & $Z=-7.350$ & 0 \\
\hline Total sleep time (min, $M \pm S D)$ & $373.9 \pm 87.4$ & & & \\
\hline Sleep latency [min, M(P25, P75)] & $30.0(15.5,59.3)$ & & & \\
\hline Sleep efficiency $(\%, M \pm S D)$ & $71.4 \pm 15.6$ & & & \\
\hline Awakening times (times, $\mathrm{M} \pm \mathrm{SD}$ ) & $18.0 \pm 6.8$ & & & \\
\hline Awakening time after sleep ( $\min , M \pm S D$ ) & $148.4 \pm 79.7$ & & & \\
\hline $\mathrm{N} 1 \%[\%, \mathrm{M}(\mathrm{P} 25, \mathrm{P} 75)]$ & $32.4(23.3,48.2)$ & & & \\
\hline $\mathrm{N} 2 \%[\%, \mathrm{M}(\mathrm{P} 25, \mathrm{P} 75)]$ & $46.2(37.9,52.2)$ & & & \\
\hline $\mathrm{N} 3 \%[\%, \mathrm{M}(\mathrm{P} 25, \mathrm{P} 75)]$ & $7.3(3.1,13.5)$ & & & \\
\hline REM\% (\%, M $\pm S D)$ & $10.1 \pm 6.7$ & & & \\
\hline
\end{tabular}

Abbreviations: MoCA-C, Beijing-Chinese Montreal Cognitive Assessment Scale; PSQI, Pittsburgh Sleep Quality Index; PSAS, Sleep Awakening Scale; REM\%, the percentage of sleep in the total sleep time during rapid eye movement; $\mathrm{N} 1 \%, \mathrm{~N} 2 \%, \mathrm{~N} 3 \%$, the percentage of non-REM sleep stages 1,2 and 3 in the total sleep time. 
patients with CID and the control group (all $P>0.05$ ), which was comparable.

\subsection{Sleep Quality}

The PSAS score of the CID group was significantly higher than that of the control group (almost twice that of the control group, $P<0.001)$. The median score of PSQI in the CID group was 16 points (indicating that the patients had moderate to severe insomnia), which was significantly higher than that in the control group $(P<0.001)$. The PSG objective sleep report in the CID group showed that the subjects' quality was ideal (in line with the change of PSG parameters of insomnia patients), and the specific results were shown in Table 1.

\subsection{Serum Copeptin and $\alpha$-Amylase Level}

The serum copeptin levels and a-amylase in CID patients were significantly higher than those in the control group (all $P<0.001$, Table 1). Among them, the levels of serum copeptin in the CID group were more than twice as high as those in the control group.

\subsection{Cognitive Function}

The MoCA-C score of the CID group was significantly lower than that of the control group (Table 1). The error numbers of object work, space work, and object recognition memory in the nine-box maze were significantly higher than those in the control group (all $P<0.05$, Table 2).

3.4. Correlation between Serum Copeptin, $\alpha-$ amylase and Clinical Parameters in CID Patients

Sleep parameters: Spearman's correlation analysis in the CID group showed that serum copeptin was positively correlated with PSAS score, while serum $\alpha$ amylase was negatively correlated with $\mathrm{N} 2 \%$, positively correlated with PSG awakening times (all $P<0.05$, Table 3).

In view that age, sex, education level, and depression had an influence on sleep and cognition, partial correlation analysis after controlling these factors showed that copeptin were positively correlated with PSQI and PSAS scores (all $P<0.05$ ), and positively correlated with sleep latency, awakening times, and $\mathrm{N} 1 \%$ of PSG sleep parameters, and negatively correlated with $\mathrm{N} 2 \%$ (all $P<0.05$ ). However, serum $\alpha$-amylase was only positively correlated with PSG awakening times $(P<0.05)$.

Further, multiple linear regression analysis, with the level of copeptin as the dependent variable and the significant factor of univariate correlation analysis as the independent variable, showed that only PSQI score and sleep latency were included into the regression equation $(P<0.05$, Table 4$)$. The regression equation was as follow:

Table 2: The Performance of Chronic Insomnia Group and Control Group in the Nine Box Maze

\begin{tabular}{|c|c|c|c|c|c|c|c|c|c|c|}
\hline \multirow{2}{*}{$\begin{array}{l}\text { Number of } \\
\text { Errors }\end{array}$} & \multicolumn{2}{|c|}{$\begin{array}{c}\text { Object Reference } \\
\text { Memory }\end{array}$} & \multicolumn{2}{|c|}{$\begin{array}{c}\text { Space Reference } \\
\text { Memory }\end{array}$} & \multicolumn{2}{|c|}{$\begin{array}{l}\text { Object Work } \\
\text { Memory }\end{array}$} & \multicolumn{2}{|c|}{$\begin{array}{l}\text { Space Work } \\
\text { Memory }\end{array}$} & \multicolumn{2}{|c|}{$\begin{array}{c}\text { Object Recognition } \\
\text { Memory }\end{array}$} \\
\hline & CID & Controls & CID & Controls & CID & Controls & CID & Controls & CID & Controls \\
\hline 0 & 54 & 29 & 50 & 28 & 26 & 20 & 5 & 13 & 41 & 28 \\
\hline 1 & 2 & 1 & 3 & 1 & 19 & 8 & 13 & 7 & 12 & 1 \\
\hline 2 & 0 & 0 & 4 & 1 & 8 & 2 & 17 & 7 & 4 & 1 \\
\hline 3 & 0 & 0 & 0 & 0 & 1 & 0 & 4 & 1 & 0 & 0 \\
\hline 4 & 1 & 0 & 0 & 0 & 3 & 0 & 11 & 2 & 0 & 0 \\
\hline 5 & 0 & 0 & 0 & 0 & 0 & 0 & 3 & 0 & 0 & 0 \\
\hline 6 & 0 & 0 & 0 & 0 & 0 & 0 & 4 & 0 & 0 & 0 \\
\hline Total & 57 & 30 & 57 & 30 & 57 & 30 & 57 & 30 & 57 & 30 \\
\hline Z-Value & \multicolumn{2}{|c|}{-0.481} & \multicolumn{2}{|r|}{-0.820} & \multicolumn{2}{|c|}{-2.099} & \multicolumn{2}{|r|}{-3.935} & \multicolumn{2}{|c|}{-2.266} \\
\hline P-Value & \multicolumn{2}{|r|}{0.676} & \multicolumn{2}{|r|}{0.412} & \multicolumn{2}{|r|}{0.036} & \multicolumn{2}{|r|}{$<0.001$} & \multicolumn{2}{|c|}{0.023} \\
\hline
\end{tabular}

Note: The data in the table are the number of subjects with various memory errors in both groups. 
Table 3: Correlations between Levels of Serum Copeptin and $\alpha$-amylase and Sleep Parameters in Chronic Insomnia Group

\begin{tabular}{|c|c|c|c|c|}
\hline \multirow{2}{*}{ Items } & \multicolumn{2}{|c|}{ Copeptin } & \multicolumn{2}{c|}{ a-amylase } \\
\cline { 2 - 5 } & $\begin{array}{c}\text { Spearman Correlation } \\
\text { Analysis }\end{array}$ & $\begin{array}{c}\text { Partial Correlation } \\
\text { Analysis }\end{array}$ & $\begin{array}{c}\text { Spearman Correlation } \\
\text { Analysis }\end{array}$ & $\begin{array}{c}\text { Partial Correlation } \\
\text { Analysis }\end{array}$ \\
\hline \hline IIIness duration (yr.) & -0.092 & -0.124 & 0.096 & 0.124 \\
\hline PSQI score & 0.186 & $0.338^{*}$ & 0.235 & 0.196 \\
\hline PSAS score & $0.332^{*}$ & $0.316^{*}$ & 0.185 & 0.106 \\
\hline Total sleep time & 0.072 & 0.063 & -0.004 & 0.018 \\
\hline Sleep latency & 0.223 & $0.324^{*}$ & 0.136 & 0.177 \\
\hline Sleep efficiency & 0.070 & 0.006 & 0.004 & 0.037 \\
\hline Awakening times & $0.328^{*}$ & $0.325^{*}$ & $0.278^{*}$ & $0.293^{*}$ \\
\hline Awakening time after & 0.048 & 0.127 & 0.060 & 0.023 \\
\hline sleep & 0.109 & $0.278^{*}$ & -0.055 & -0.050 \\
\hline N1\% & -0.105 & $-0.279^{*}$ & $-0.263^{*}$ & -0.214 \\
\hline N2\% & -0.227 & -0.220 & 0.225 & 0.204 \\
\hline N3\% & 0.083 & -0.111 & 0.149 & -0.125 \\
\hline REM\% & & & \\
\hline
\end{tabular}

Note: The values in the table are correlation coefficients, and * means $P<0.05$;

Abbreviations: PSQI, Pittsburgh Sleep Quality Index; PSAS, Sleep Awakening Scale; REM\%, the percentage of sleep in the total sleep time during rapid eye movement; $\mathrm{N} 1 \%, \mathrm{~N} 2 \%, \mathrm{~N} 3 \%$, The percentage of non-REM sleep stage 1, 2 and 3 in the total sleep time.

$y=2.199 x+1.06, y=0.164 x+0.079$

Cognitive parameters: The levels of serum copeptin and $\alpha$-amylase in CID patients were not significantly correlated with the MoCA-C score and the numbers of memory errors related to the Nine-Box Maze.

\section{DISCUSSION}

Neuroelectrophysiological study found that the slow wave sleep power spectrum of the CID patients had certain changes, suggesting that there was an enhanced degree of cortical activity. Monitoring of autonomic nervous function, such as heart rate variability, skin electrical stimulation and temperature fluctuations, found that insomnia patients had significantly increased sympathetic activity and high arousal at night. Chronic insomnia act as a chronic stressor to cause neuroendocrine disorders, directly and/or indirectly affects the quality of sleep, forming a vicious circle [3]. Recent studies [19] have shown that chronic insomnia is mainly accompanied by increased

Table 4: Regression Analysis of Influencing Factors for Copeptin Level

\begin{tabular}{|c|c|c|c|}
\hline Variables & $\boldsymbol{\beta}$ values & $\boldsymbol{t}$ values & $\boldsymbol{P}$ values \\
\hline \hline PSQI score & 0.255 & 2.075 & $0.043^{*}$ \\
\hline PSAS score & 0.170 & 1.365 & 0.178 \\
\hline a-amylase & -0.213 & -1.597 & 0.117 \\
\hline Sleep latency & 0.54 & 2.076 & $0.043^{*}$ \\
\hline Awakening times & 0.217 & 1.681 & 0.099 \\
\hline N1\% & -0.019 & -0.097 & 0.923 \\
\hline N2\% & 0.213 & -1.106 & 0.274 \\
\hline
\end{tabular}

Note: ${ }^{*} P<0.05$

Abbreviations: PSQI, Pittsburgh Sleep Quality Index; PSAS, Sleep Awakening Scale; N1\%, N2\%, the percentage of non-rapid eye movement sleep stages 1 and stage 2 in the total sleep time. 
sympathetic nerve activity. However, the correlation between sympathetic hyperfunction and CID remains unclear.

AVP is mainly produced by the hypothalamic paraventricular nucleus and regulated by various neurons from different brain areas [9]. AVP is a sympathetically activated effector, and its neurons can receive excitatory impulses transmitted from noradrenergic neurons in locus coeruleus during stress [20]. Many studies have shown that AVP is paramount in regulating the cardiovascular system, endocrine system, anxiety, depression, other physical diseases, and behavior and memory [21]. As the carboxylterminal fragments of AVP, copeptin release equimolar as AVP and are more stable in vivo. Therefore, copeptin can be used as an equivalent indicator of AVP concentration [10]. We studied the changes in serum and copeptin levels in CID patients, and the results showed a significant increment of the serum copeptin level in the CID patients, indicating that the sufferers had sustained sympathetic hyperfunction under longterm stress. Our result supported the viewpoint of sympathetic hyperactivity in CID derived from the previous studies about skin temperature and heart-rate variability [22, 23]. Surprisingly, there was no correlation between copeptin level and illness duration after adjusting age, sex, education level, and other confounding factors. The reason might be that with the prolongation of stress duration, sympathetic activity achieve a plateau stage and then does not change with time anymore.

As we all know, CID patients mostly have bad cognition and negative emotions before sleeping. Under the combination of physiological, cognitive, and emotional characteristics of high arousal as well as presleep high awakening state, the occurrence and maintenance of insomnia always be promoted [24]. In this study, the PSAS score of CID patients was significantly increased, which indicated that CID patients were highly susceptible to insomnia and had pre-sleep cognitive and physical hyperarousal [16]. As it was predicted, our results also showed that the level of copeptin in CID patients was positively correlated with the PSAS score. These findings indicated that sympathetic nerve activity may play an important role in the pre-sleep high arousal state.

The relationship between sympathetic nerve activity and sleep quality parameters has not yet been studied so far. Among the previous studies evaluating CID patients' sympathetic activity (such as heart rate, blood/urine catecholamine level), few studies have reported the correlation between sympathetic activity and sleep parameters [19]. The results of correlation analysis in our study showed that the higher the serum copeptin level of CID patients, the worse the subjective sleep quality (which can be seen from the positive correlation with PSQI score), and the objective sleep quality was characterized by prolonged sleep latency, increased awake times at night, increased shallow sleep and decreased deep sleep. The above results enriched the theoretical understanding of the connection between insomnia and sympathetic nerve activity. We hypothesized that the increase of sympathetic nerve excitability in CID patients would lead to the change of sleep structure and the decline of sleep quality, and eventually result in the occurrence and maintenance of insomnia [25]. Further regression analysis showed that there was a significant correlation between copeptin and PSQI score and sleep latency, indicating that the hyperactive sympathetic nerves contribute to the high awakening state that readily induce insomnia, making it more difficult to fall asleep [26]. Above results indicated that copeptin is more likely to be correlated with the CID subtype in the sufferers characterized by sleep initiating difficulty. This concept could be enhanced by the findings come from another study [27] which has shown that the sleep latency of insomnia patients with high sleep response is prolonged.

Alpha-amylase is mainly secreted by pancreatic acinar cells, and its production and secretion are regulated by the autonomic nervous system. The activation of sympathetic nerves will increase the secretion of $\alpha$-amylase [28]. Our study showed that the serum a-amylase level in CID patients was significantly increased, which provided another evidence of sympathetic hyperactivity. However, our study found that serum $\alpha$-amylase was only positively correlated with the waking frequency of CID patients. Above results indicated that copeptin may be correlated with the whole process of insomnia, specifically difficulty in sleep initiation, while $\alpha$-amylase may be correlated with the difficulty in sleep maintenance. However, its specific mechanism remains to further be resrached.

Most people have recognized the view that good sleep contributes to memory consolidation, and our study also confirmed that CID patients suffer from impairment of object working memory, spatial working memory, and object recognition memory, which was consistent with our previous research results [21]. Our previous study [29] also suggested that impaired 
microstructure of the brain may be associated with these damaged memory systems in the CID patients, but the current study did not found and correlation between copeptin and $\alpha$-amylase levels and cognitive impairment. The reason might be that the sympathetic activity is mainly response for those autonomic functions (such as activity of blood vessels, heartbeat, sweat), and a proper increase of sympathetic nerve activity can enhance cognitive function. The specific mechanism underlying above phenomenon needs further to be explored.

In conclusion, our study showed that CID patients had significant sympathetic hyperactivity, which might be linked to the difficulty in both initiation and maintenance of sleep in the CID, especially the copeptin, which provided a new idea for clinical prevention and treatment of insomnia. In the follow-up study, it is necessary to expand the sample size, combine with other variables (such as heart rate variability and cortisol), increase the comparison of indicators before and after treatment to further explore the influence of sympathetic nerve activity on sleep and cognitive function in patients with chronic insomnia.

\section{FUND PROGRAM}

General Program of National Natural Science Foundation of China (81671316); Natural Science Foundation of Anhui Medical University (2018xk1066)

\section{CONFLICT OF INTEREST}

All authors declare that there is no conflict of interest.

\section{CONTRIBUTION OF AUTHOR}

Ting Hu: Clinical data and blood sample collection, data processing, and writing the first draft of the article;

Xuan Song: Collection and analysis of clinical data, literature review;

Yi-Jun Ge: Assisting in data analysis and paper writing guidance;

Ping Zhang: Statistical analysis; paper revision

Xue-Yan Li, Xiao-Yi Kong: Provide case information;

Gui-Hai Chen: Proposing research ideas, paper revision, and overall guidance.

\section{REFERENCES}

[1] Chen GH and Zhao ZX. Insomnia. In: Zhao ZX editor-inchief. Sleep medicine [M]. First edition, Beijing, people's health publishing house 2016; 83-88.

[2] Pang R, Guo R, Wu X, et al. Altered regional homogeneity in chronic insomnia disorder with or without cognitive impairment. AJNR Am J Neuroradiol 2018; 39(4): 742-747. https://doi.org/10.3174/ajnr.A5587

[3] Bianchi MT. Chronic Insomnia. Semin Neurol. 2017; 37(4): 433-438. https://doi.org/10.1055/s-0037-1605344

[4] Riemann D, Spiegelhalder K, Feige B, et al. The hyperarousal model of insomnia: a review of the concept and its evidence. Sleep Med Rev 2010; 14(1): 19-31. https://doi.org/10.1016/j.smrv.2009.04.002

[5] Tobaldini E, Costantino G, Solbiati M, et al. Sleep, sleep deprivation, autonomic nervous system and cardiovascular diseases. Neurosci Biobehav Rev 2017; 74(Pt B): 321-329. https://doi.org/10.1016/j.neubiorev.2016.07.004

[6] Zhao ZQ, Li YP, Xiang XX, et al. Pathophysiology and cognitive behavioral therapy of chronic insomnia[J]. Chin $J$ Clin Pharmacol Ther, 2021; 26(5): 481-486.

[7] Xia L, Chen GH, Li ZH, et al. Alterations in hypothalamuspituitary-adrenal/thyroid axes and gonadotropin-releasing hormone in the patients with primary insomnia: a clinical research. PLoS One 2013; 8 (8): e71065. https://doi.org/10.1371/journal.pone.0071065

[8] Thomson F, Craighead M. Innovative approaches for the treatment of depression: targeting the HPA axis. Neurochem Res 2008; 33(4): 691-707. https://doi.org/10.1007/s11064-007-9518-3

[9] Ozben S, Guvenc TS, Huseyinoglu N, et al. Low serum copeptin levels in patients with obstructive sleep apnea. Sleep Breath, 2013, 17(4): 1187-1192. https://doi.org/10.1007/s11325-013-0822-7

[10] Christ-Crain M. Vasopressin and copeptin in health and disease. Rev Endocr Metab Disord 2019; 20(3): 283-294. https://doi.org/10.1007/s11154-019-09509-9

[11] Çınarka $H$, Kayhan $S$, Karataş $M$, et al. Copeptin: a new predictor for severe obstructive sleep apnea. Ther Clin Risk Manag 2015; 11: 589-594. https://doi.org/10.2147/TCRM.S80779

[12] Bosch JA, Veerman EC, de Geus EJ, Proctor GB. a-Amylase as a reliable and convenient measure of sympathetic activity: don't start salivating just yet!. Psychoneuroendocrinology 2011; 36(4): 449-453.

https://doi.org/10.1016/j.psyneuen.2010.12.019

[13] Sateia MJ. International classification of sleep disorders-third edition: highlights and modifications. Chest 2014; 146(5): 1387-1394.

https://doi.org/10.1378/chest.14-0970

[14] Buysse DJ, Reynolds CF 3rd, Monk TH, et al. The Pittsburgh Sleep Quality Index: a new instrument for psychiatric practice and research. Psychiatry Res 1989; 28(2): 193-213. https://doi.org/10.1016/0165-1781(89)90047-4

[15] Hamilton M. A rating scale for depression. J Neurol Neurosurg Psychiatry 1960; 23: 56-62. https://doi.org/10.1136/jnnp.23.1.56

[16] Nicassio PM, Mendlowitz DR, Fussell JJ, et al. The phenomenology of the pre-sleep state: the development of the pre-sleep arousal scale. Behav Res Ther 1985; 23(3): 263- 271.

https://doi.org/10.1016/0005-7967(85)90004-X

[17] Chen $X$, Zhang R, Xiao $Y$, et al. Reliability and validity of the Beijing version of the Montreal Cognitive Assessment in the evaluation of cognitive function of adult patients with OSAHS. PLoS One 2015; 10(7): e0132361.

https://doi.org/10.1371/journal.pone.0132361 
[18] Wei QG, Chen GH, Xia L, et al. The differences of memories and sleep parameters and their correlations in patients with insomnia disorder in different subtypes: a clinical study. Chin J Neurol 2015; 48(9): 763-767.

[19] Grimaldi D, Goldstein MR, Carter JR. Insomnia and cardiovascular autonomic control. Auton Neurosci 2019; 220: 102551.

https://doi.org/10.1016/j.autneu.2019.05.003

[20] Schinke C, Hesse S, Rullmann M, et al. Central noradrenaline transporter availability is linked with HPA axis responsiveness and copeptin in human obesity and non-obese controls. Stress 2019; 22(1): 93-102. https://doi.org/10.1080/10253890.2018.1511698

[21] Szczepanska-Sadowska E, Zera T, Sosnowski P, et al. Vasopressin and related peptides; potential value in diagnosis, prognosis and treatment of clinical disorders. Curr Drug Metab 2017; 18(4): 306-345. https://doi.org/10.2174/1389200218666170119145900

[22] Gu X, Gu P, Liu YH, et al. Correlation of sleep quality, anxiety, depression and sympathetic skin response in chronic insomnia. Chin J Neurol 2017; 50(9): 665-670.

[23] Carter JR, Grimaldi D, Fonkoue IT, et al. Assessment of sympathetic neural activity in chronic insomnia: evidence for elevated cardiovascular risk. Sleep 2018; 41(9): zsy126. https://doi.org/10.1093/sleep/zsy126

[24] Jarrin DC, Chen IY, Ivers H, et al. The role of vulnerability in stress-related insomnia, social support and coping styles on incidence and persistence of insomnia. J Sleep Res 2014;
23(6): 681-688.

https://doi.org/10.1111/jsr.12172

[25] Palagini L, Moretto $U$, Novi M, et al. Lack of resilience is related to stress-related sleep reactivity, hyperarousal, and emotion dysregulation in insomnia disorder. J Clin Sleep Med 2018; 14(5): 759-766. https://doi.org/10.5664/jcsm.7100

[26] Lozić M, Šarenac O, Murphy D, et al. Vasopressin, central autonomic control and blood pressure regulation. Curr Hypertens Rep 2018; 20(2): 11.

https://doi.org/10.1007/s11906-018-0811-0

[27] Gehrman PR, Hall M, Barilla $\mathrm{H}$, et al. Stress reactivity in insomnia. Behav Sleep Med 2016; 14(1): 23-33. https://doi.org/10.1080/15402002.2014.940112

[28] Nater UM, Rohleder N. Salivary alpha-amylase as a non-invasive biomarker for the sympathetic nervous system: current state of research. Psychoneuroendocrinology 2009; 34(4): 486-496. https://doi.org/10.1016/j.psyneuen.2009.01.014

[29] Zhang P, Tan CW, Chen GH, et al. Patients with chronic insomnia disorder have increased serum levels of neurofilaments, neuron-specific enolase and S100B: does organic brain damage exist? Sleep Med 2018; 48: 163-171. https://doi.org/10.1016/j.sleep.2017.12.012

Received on 12-11-2021

Accepted on 26-11-2021

Published on 06-12-2021

http://dx.doi.org/10.15379/2409-3564.2021.08.02.01

(c) 2021 Hu et al.; Licensee Cosmos Scholars Publishing House.

This is an open access article licensed under the terms of the Creative Commons Attribution Non-Commercial License

(http://creativecommons.org/licenses/by-nc/3.0/), which permits unrestricted, non-commercial use, distribution and reproduction in any medium, provided the work is properly cited. 